\title{
Phân tích rủi ro trong giai đoạn chuẩn bị dự án nhà cao tầng của chủ đầu tư tại Tp.Hồ Chí Minh
}

\author{
Hoàng Văn Dương ${ }^{1 *}$, Lương Đức Long ${ }^{1}$ \\ ${ }^{1}$ Khoa Kỹ Thuật Xây Dựng, Trường Đại Học Bách Khoa - Đại Học Quốc Gia Tp.HCM

TƯ KHOÁ
Phân tích rủi ro xây dựng
Rủi ro trong giai đoạn chuẩn bị
Rủi ro trong giai đoạn nghiên
cứu khả thi \\ TÓM TẮT \\ Vòng đời của tất cả các dự án (Bao gồm dự án đầu tư xây dựng) đều chứa đựng các rủi ro. Trong giai \\ đoạn chuẩn bị dự án, có rất nhiều rủi ro tiềm ẩn, gây hậu quả nghiêm trọng làm ảnh hưởng các giai \\ đoạn sau và toàn dự án, song thực tế các nghiên cứu về rủi ro trong giai đoạn chuẩn bị dự án còn rất \\ hạn chế so với các nghiên cứu liên quan khác. Bài báo này tập trung phân tích các nhân tố rủi ro ảnh \\ hưởng trực tiếp đến tiến độ và chi phí trong giai đoạn chuẩn bị dự án. Thực hiện khảo sát 28 nhân tố \\ rủi ro được chia thành 05 nhóm chính: Tài chính và Kinh tế (1); Chính sách và Pháp luật (2); Kỹ thuật \\ và Công nghệ (3); Tổ chức và Quản lý (4); Môi trường và Xã hội (5). Bảng khảo sát được thu thập và \\ lấy ý kiến từ các chuyên gia, các nhân sự có nhiều kinh nghiệm làm việc cho các công ty xây dựng tại \\ Tp.HCM để tìm ra các nhân tố rủi ro xảy ra thường xuyên và có mức độ tác động lớn, ảnh hưởng trong \\ giai đoạn. Từ đó cung cấp cho các doanh nghiệp, các Chủ đầu tư có kế hoạch điều chỉnh đường lối, \\ chính sách phù hợp nhằm nâng cao hiệu quả trong việc đầu tư và quản lý dự án.
}

\section{KEYWORDS}

Risks analysis in construction Risks in the preparation phase Risk in feasibility study Risk response strategies Severity of the risk

\begin{abstract}
The life cycle of all projects (including construction investment projects) contains risks. In the preparation phase of the project, there are many potential risks, causing serious consequences that affect the following stages and the whole project, but in fact, the research on risks in the preparation phase project implementation is still very limited compared to other related studies. This paper focuses on analyzing the risk factors that directly affect the progress and costs in the preparation phase of the project. A survey of 28 risk factors divided into 5 main groups: Finance and Economics (1); Policy and Law (2); Engineering and Technology (3); Organization and Management (4); Environment and Society (5). The survey was collected and consulted from experts and experienced personnel working for construction companies in HCM City to find out the risk factors that occur frequently and and have a large degree of influence during this period. Thereby, it will provide businesses and owners with appropriate plans to adjust guidelines and policies to improve efficiency in investment and project management.
\end{abstract}

\section{Giới thiệu}

Ngành xây dựng là một ngành nghề có lịch sử phát triển lâu đời và được phổ biến rộng khắp trên toàn thế giới. Xu hướng toàn cầu hóa đang đóng vai trò chủ đạo khi các Nhà đầu tư luôn tìm kiếm những môi trường mới nổi để đa dạng hóa các sản phẩm, mở rộng các hoạt động kinh doanh và giảm thiểu tối đa các rủi ro tiềm ẩn. Với điều kiện kinh tế tương lai thuận lợi, Chủ đầu tư dự án xây dựng ngày càng có sự chuyên môn hóa theo các loại hình sản phẩm đầu ra nhằm tối ưu hóa lợi nhuận.

Thực tế hiện nay các doanh nghiệp tư nhân đầu tư xây dựng không ngừng phát triển về số lượng và quy mô, tuy nhiên việc nhận dạng, đánh giá, ứng phó các rủi ro trong giai đoạn chuẩn bị lại không được các doanh nghiệp chú trọng, thậm chí còn đối phó rất bị động, chính các tác động rủi ro này dẫn đến việc thay đổi nhiều kế hoạch và dự tính ban đầu, làm thay đổi hiệu quả đầu tư của dự án, kéo dài thời gian thực hiện và phát sinh chi phí. Trường hợp các yếu tố rủi ro xảy ra, đó không chỉ đơn thuần là việc gây ra ảnh hưởng nội tại đối với chính công tác đó mà còn làm ảnh hưởng đến toàn bộ dự án về các khía cạnh như: Tiến độ, chi phí, chất lượng... Việc chủ động nhận dạng và có các biện pháp ứng phó rủi ro trong giai đoạn chuẩn bị thực hiện là vô cùng cần thiết nhằm đảm bảo tính khả thi và hiệu quả cho dự án. 
Thông qua bài báo này, tác giả sẽ trình bày nghiên cứu để tìm ra những nhân tố rủi ro thường xuyên xảy ra và có mức độ ảnh hưởng nghiêm trọng đến hiệu quả dự án đầu tư xây dựng, qua đó mong muốn đóng góp một phần cơ sở dữ liệu nhằm đánh giá các yếu tố rủi ro trong giai đoạn chuẩn bị dự án, từ đó đề xuất các ứng phó, xây dựng quy trình làm việc hiệu quả, nâng cao hiệu quả quản lý của Chủ đầu tư đối với các dự án đầu tư xây dựng nhà cao tầng sử dụng vốn tư nhân.

\section{Tổng quan về nghiên cứu}

Rủi ro là cơ hội xảy ra của cái gì đó mà gây ra tác động vào các mục tiêu của dự án [1]. Rủi ro (hay cơ hội) là cái gây ra kết quả bất lợi hoặc thuận lợi cho các mục tiêu của dự án [2]. Rủi ro trong dự án xây dựng có thể chia ra làm 02 loại: Rủi ro (rủi ro gây hại) và Cơ hội (rủi ro có ích). Theo PMBOK [3] thì quản lý rủi ro là tập hợp các công việc liên quan đến nhân dạng, phân tích đánh giá và phản ứng lại sự không chắc chắn xuyên suốt vòng đời của một dự án. Về cơ bản, các thành phần của rủi ro bao gồm:

Tính chất của sự kiện (xấu hay tốt).

Khả năng (xác suất, cơ hội) xảy ra sự kiện đó.

Hậu quả (tác động) của sự kiện đó.

Khoảng thời gian (thời điểm) xảy ra sự kiện đó.

Hiện nay, các nghiên cứu về rủi ro trong xây dựng là rất đa dạng và phổ biến. Tại các nước phát triển, quản lý rủi ro là yêu cầu bắt buộc trong việc quản lý dự án, là chìa khóa quyết định thành công. Tại các nước đang phát triển, quản lý rủi ro còn dừng ở mức nhận thức, việc ứng phó rủi ro còn bó hẹp trong phạm vi hợp đồng và chưa ứng dụng nhiều vào các hoạt động thực tiễn của dự án [4]. Các nghiên cứu liên quan rủi ro trong giai đoạn chuẩn bị dự án trên Thế giới nói chung và tại Việt Nam nói riêng là rất ít và hạn chế, các nghiên cứu chỉ tập trung vào việc phân tích và đánh giá hiệu quản dự án. Từ thực trạng trên có thể thấy, sự quan tâm về các kết quả và ứng phó rủi ro trong giai đoạn chuẩn bị thực hiện của Chủ đầu tư chưa thực sự được chú trọng. Công tác quản lý rủi ro còn rời rạc, không kết nối xây dựng được các quy định, các yêu cầu chung về việc kiểm soát và quản lý rủi ro.

Nghiên cứu của Fangfang Wen và Ling Li (2013) [5] , "Nghiên cứu về rủi ro đầu tư dự án Bất động sản bằng phương pháp AHP-fuzzy". Kết quả nghiên cứu đã chỉ ra 7 nhóm yếu tố rủi ro chính bao gồm: Rủi ro chính sách, Rủi ro thị trường, Rủi ro kinh tế, Rủi ro tài chính, Rủi ro chi phí, Rủi ro marketing, Rủi ro dịch vụ quản lý khách hàng”, theo tác giả đây là các nhóm rủi ro chính ảnh hưởng đến hiệu quả đầu tư xây dựng. Kết quả nghiên cứu các rủi ro liên quan đến thị trường và kinh tế được quan tâm nhiều, thông qua đó đề xuất cải tiến hệ thống kiểm soát nội bộ và thực hiện các biện pháp hữu hiệu để tránh rủi ro hoặc giảm khả năng xảy ra rủi ro, từ đó đảm bảo thành công chung cho dự án.
Nghiên cứu của Bayu Aditya Firmansyah và cộng sự (2006) [6], "Phân tích rủi ro trong giai đoạn nghiên cứu khả thi dự án công trình xây dựng: Case study - Pt. Perusahaan gas negara Indonesia”. Kết quả nghiên cứu đã chỉ ra 8 nhóm yếu tố rủi ro chính bao gồm: Rủi ro kinh doanh, marketing; Rủi ro kỹ thuật, công nghệ; Rủi ro chính trị; Rủi ro về quy định, chính sách; Rủi ro văn hóa xã hội; Rủi ro môi trường và không gian quy hoạch; Rủi ro tài chính; Rủi ro kinh tế. Kết quả nghiên cứu, xếp hạng hàng đầu là các biến rủi ro có mối quan hệ với khía cạnh kinh tế và tài chính. Điều này cho thấy rằng kinh tế rất dễ thay đổi, các sự thay đổi của các yếu tố kinh tế có ảnh hưởng trực tiếp đến lợi ích mà các nhà đầu tư sẽ đạt được.

Tại Việt Nam, nghiên cứu của Nguyễn Quốc Toản (2019) [7] về quản lý rủi ro: Bài học kinh nghiệm và giải pháp trong giai đoạn chuẩn bị dự án - Trường hợp khảo sát tại Ban quản lý dự án Thành phố Uông Bí, Quảng Ninh. Kết quả nghiên cứu chỉ ro các rủi ro gặp phải trong giai đoạn chuẩn bị dự án bao gồm: Dự báo và các thông tin ban đầu chưa chính xác; Sự không phù hợp giữa mục tiêu của dự án và tình hình thực tế; Lựa chọn địa điểm xây dựng; Lựa chọn kỹ thuật và công nghệ; Lựa chọn phương án nguồn vốn, Các thủ tục hành chính, pháp lý. Tác giả đã phân tích cụ thể các rủi ro tại dự án, từ đó đề xuất phương pháp phòng tránh rủi ro và giải pháp hoàn thiện.

Hay nghiên cứu của Lê Văn Long (2006) [8]. Một số vấn đề quản lý rủi ro trong dự án đầu tư xây dựng công trình. Tác giả đã đề cập rủi ro trong giai đoạn chuẩn bị dự án thường liên quan đến thông tin dữ liệu điều tra phục vụ tính toán dự án, thời gian thực hiện quá trình chuẩn bị dự án, căn cứ pháp lý của dự án. Từ đó xây dựng quá trình quản lý rủi ro dự án đầu tư xây dựng công trình.

\section{Phương pháp nghiên cứu}

Nghiên cứu được thực hiện thông qua việc tham khảo các tài liệu, đề tài tương tự, tham khảo ý kiến của các chuyên gia trong lĩnh vực liên quan có hiểu biết và quan tâm về rủi ro trong giai đoạn chuẩn bị dự án. Sử dụng thang đo Likert 5 mức độ để đánh giá Khả năng xảy ra và Mức độ tác động đến tiêu chí Tiến độ và Chi phí.

\section{Bảng 1.}

Mã hóa các biến rủi ro trong giai đoạn chuẩn bị dự án.

\begin{tabular}{|c|c|c|c|c|}
\hline Stt & Ký hiệu & \multicolumn{1}{|c|}{ Các yếu tố rủi ro } & \multicolumn{2}{|c|}{ Mã biến } \\
\cline { 3 - 5 } & Chi phí & Tiến độ \\
\hline \multicolumn{2}{|c|}{ A. Nhóm Tài chính và Kinh tế } \\
\hline 1 & $\mathrm{~A} 1$ & $\begin{array}{l}\text { Nghiên cứu, dự báo nhu cầu thị } \\
\text { trường không chính xác }\end{array}$ & CPA1 & TDA1 \\
\hline 2 & A2 & $\begin{array}{l}\text { Định hướng sản phẩm kinh } \\
\text { doanh và tính chất của khu đất } \\
\text { không phù hợp }\end{array}$ & CPA2 & TDA2 \\
\hline
\end{tabular}




\begin{tabular}{|c|c|c|c|c|}
\hline \multirow{2}{*}{ Stt } & \multirow{2}{*}{ Ký hiệu } & \multirow{2}{*}{ Các yếu tố rủi ro } & \multicolumn{2}{|c|}{ Mã biến } \\
\hline & & & Chi phí & Tiến độ \\
\hline 3 & A3 & $\begin{array}{l}\text { Thiếu hụt nguồn vốn, khả năng huy } \\
\text { động vốn không tốt }\end{array}$ & CPA3 & TDA3 \\
\hline 4 & A4 & $\begin{array}{l}\text { Tính toán các chỉ tiêu tài chính sai } \\
\text { sót và chưa phù hợp với thực tế }\end{array}$ & CPA4 & TDA4 \\
\hline 5 & A5 & $\begin{array}{l}\text { Biến động nền kinh tế thị trường, } \\
\text { tốc độ tăng trưởng chậm }\end{array}$ & CPA5 & TDA5 \\
\hline 6 & A6 & Lãi suất các bên cho vay tăng cao & CPA6 & TDA6 \\
\hline 7 & A7 & Xung đột lợi ích từ các bên đầu tư & CPA7 & TDA7 \\
\hline 8 & A8 & Lạm phát tăng & CPA8 & TDA8 \\
\hline \multicolumn{5}{|c|}{ B. Chính sách và Pháp luật } \\
\hline 1 & B1 & $\begin{array}{l}\text { Vướng công tác đền bù, giải phóng } \\
\text { mặt bằng }\end{array}$ & CPB1 & TDB1 \\
\hline 2 & B2 & $\begin{array}{l}\text { Vướng quy hoạch tổng mặt bằng, } \\
\text { quy hoạch chi tiết }\end{array}$ & CPB2 & TDB2 \\
\hline 3 & B3 & $\begin{array}{l}\text { Điều chỉnh và phê duyệt hồ sơ đầu } \\
\text { tư, hồ sơ thiết kế kéo dài, nhiều thủ } \\
\text { tục phát sinh từ Cơ quan quản lý } \\
\text { Nhà nước }\end{array}$ & CPB3 & TDB3 \\
\hline 4 & B4 & $\begin{array}{l}\text { Các chính sách, hỗ trợ của Chính } \\
\text { phủ không minh bạch }\end{array}$ & CPB4 & TDB4 \\
\hline 5 & B5 & $\begin{array}{l}\text { Khó khăn khi xin cấp phép xây } \\
\text { dựng }\end{array}$ & CPB5 & TDB5 \\
\hline \multicolumn{5}{|c|}{ C. Kỹ thuật và công nghệ } \\
\hline 1 & $\mathrm{C} 1$ & $\begin{array}{l}\text { Công tác khảo sát địa hình, địa chất } \\
\text { gặp sai sót }\end{array}$ & CPC1 & TDC1 \\
\hline 2 & C2 & $\begin{array}{l}\text { Phương án thiết kế ý tưởng chưa } \\
\text { hợp lý }\end{array}$ & СРC2 & TDC2 \\
\hline 3 & C3 & $\begin{array}{l}\text { Lựa chọn kỹ thuật, công nghệ } \\
\text { không phù hợp với tính chất dự án }\end{array}$ & CPC3 & TDC3 \\
\hline 4 & $\mathrm{C} 4$ & $\begin{array}{l}\text { Điều kiện triển khai và quy mô mức } \\
\text { độ phức tạp của các dự án đầu tư } \\
\text { xây dựng }\end{array}$ & CPC4 & TDC4 \\
\hline 5 & C5 & $\begin{array}{l}\text { Năng lực các đơn vị Tư vấn thiết } \\
\text { kế, Tư vấn lập đầu tư và Ban Quản } \\
\text { lý dự án không đáp ứng yêu cầu }\end{array}$ & CPC5 & TDC5 \\
\hline \multicolumn{5}{|c|}{ D. Tổ chức và Quản lý } \\
\hline 1 & D1 & $\begin{array}{l}\text { Mục tiêu đầu tư, phương án đầu tư } \\
\text { của dự án không phù hợp với tình } \\
\text { hình thực tế }\end{array}$ & CPD1 & TDD1 \\
\hline 2 & D2 & Thay đổi cơ cấu tổ chức & CPD2 & TDD2 \\
\hline 3 & D3 & $\begin{array}{l}\text { Cấu trúc, sơ đồ tổ chức phức tạp, } \\
\text { chồng chéo }\end{array}$ & CPD3 & TDD3 \\
\hline
\end{tabular}

\begin{tabular}{|c|c|c|c|c|}
\hline \multirow{2}{*}{ Stt } & \multirow{2}{*}{ Ký hiệu } & \multirow{2}{*}{ Các yếu tố rủi ro } & \multicolumn{2}{|c|}{ Mã biến } \\
\hline & & & Chi phí & Tiến độ \\
\hline 4 & D4 & $\begin{array}{l}\text { Các bên phối hợp, giao tiếp thông } \\
\text { tin không hiệu quả }\end{array}$ & CPD4 & TDD4 \\
\hline 5 & D5 & $\begin{array}{l}\text { Quản lý các hợp đồng Tư vấn kém } \\
\text { (Tư vấn: Khảo sát, Lập báo cáo } \\
\text { nghiên cứu khả thi, Thiết kế, Giám } \\
\text { sát...) }\end{array}$ & CPD5 & TDD5 \\
\hline \multicolumn{5}{|c|}{ E. Môi trường và Xã hội } \\
\hline 1 & E1 & $\begin{array}{l}\text { Các vấn đề về ô nhiễm môi trường } \\
\text { (Nước thải, khói bụi, tiếng ồn...) }\end{array}$ & CPE1 & TDE1 \\
\hline 2 & E2 & $\begin{array}{l}\text { An ninh và tình trạng xã hội ở dự } \\
\text { án }\end{array}$ & CPE2 & TDE2 \\
\hline 3 & E3 & $\begin{array}{l}\text { Kế hoạch đầu tư ảnh hưởng đến lợi } \\
\text { ích của tập thể, cá nhân xung } \\
\text { quanh }\end{array}$ & CPE3 & TDE3 \\
\hline 4 & E4 & $\begin{array}{l}\text { Các vấn đề liên quan đến điều kiện } \\
\text { làm việc, sức khỏe, an toàn lao } \\
\text { động và vệ sinh môi trường }\end{array}$ & CPE4 & TDE4 \\
\hline 5 & E5 & $\begin{array}{l}\text { Rủi ro bất khả kháng như dịch } \\
\text { bệnh Covid, thiên tai, biến đồi khí } \\
\text { hậu }\end{array}$ & CPE5 & TDE5 \\
\hline
\end{tabular}

\subsection{Quy trình nghiên cứu}

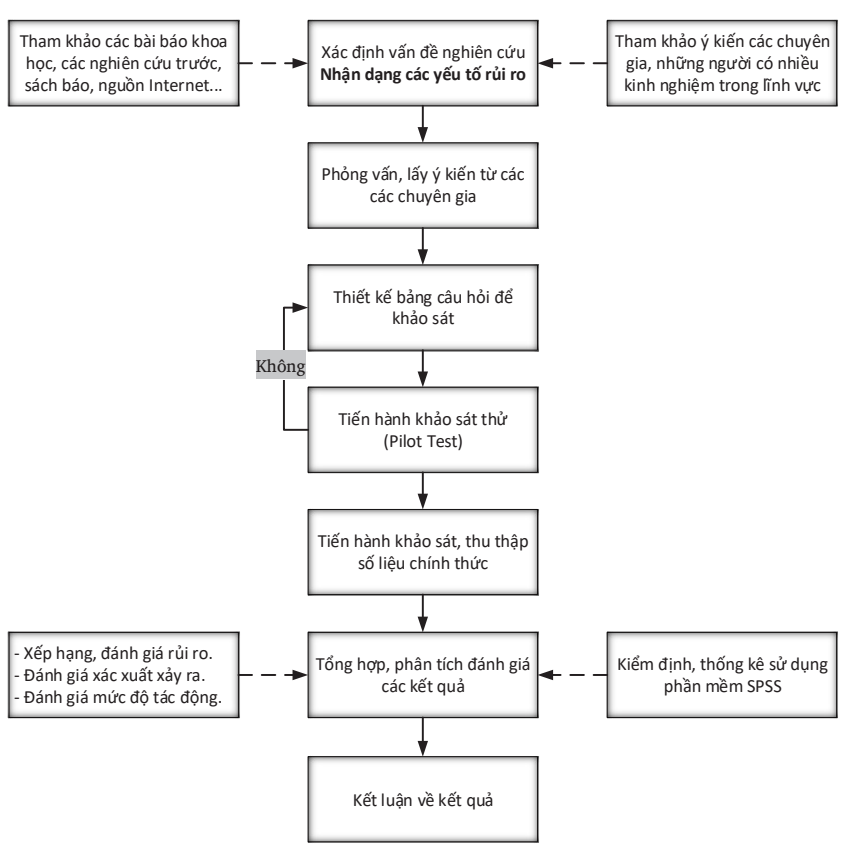

Hình 1. Quy trình nghiên cứu. 


\subsection{Công cu hỗ trơ nghiên cứu}

\section{Bảng 2.}

Công cụ hỗ trợ nghiên cứu.

\begin{tabular}{|c|l|l|}
\hline Stt & \multicolumn{1}{|c|}{ Nội dung nghiên cứu } & \multicolumn{1}{|c|}{ Công cụ hỗ trợ nghiên cứu } \\
\hline 1 & Xác định các yếu tố rủi ro của Chủ đầu tư trong giai đoạn chuẩn bị đầu tư dự án nhà cao tầng & Bảng câu hỏi \\
\hline 2 & Mô tả dữ liệu & Thống kê mô tả \\
\hline 3 & Xếp hạng các chỉ số & $\begin{array}{l}\text { Trị trung bình } \\
\text { (Phần mềm SPSS) }\end{array}$ \\
\hline 4 & Kiểm tra độ tin cậy của thang đo & $\begin{array}{l}\text { Hệ số Conbach's Alpha } \\
\text { (Phần mềm SPSS) }\end{array}$ \\
\hline 5 & Xác định yếu tố quan trọng & $\begin{array}{l}\text { Phân tích thành tố EFA } \\
\text { (Phần mềm SPSS ) }\end{array}$ \\
\hline 6 & Vẽ quy trình, sơ đồ & Phần mềm Visio \\
\hline 7 & Lập bảng tính, tiến độ & Phần mềm Excell và MS Project \\
\hline
\end{tabular}

\subsection{Thu thập số liệu}

Bảng câu hỏi được gửi đi và nhận phản hồi từ tháng 03 đến tháng 04 năm 2021, đối tượng khảo sát là các chuyên gia, chủ đầu tư, ban quản lý dự án, kiến trúc sư, kỹ sư, học viên Cao học trường Đại học Bách Khoa TP. HCM và các cá nhân, tổ chức hoạt động trong lĩnh vực xây dựng có hiểu biết về rủi ro trong giai đoạn chuẩn bị dự án nhà cao tầng trên địa bàn thành phố Hồ Chí Minh. Số lượng bảng câu hỏi phát ra là 201 bảng và thu về được 151 bảng (chiếm 75 \%) trong đó có 41 bảng không hợp lệ (chiếm 20 \%) bị loại ra. Số bảng hợp lệ là 110 bảng (chiếm $55 \%)$.

\section{Kết quả nghiên cứu và phân tích số liệu}

\subsection{Tổng hợp thông tin đối tượng khảo sát}

\section{Bảng 3.}

Tổng hợp thông tin đối tượng khảo sát.

\begin{tabular}{|c|c|c|c|c|c|c|c|}
\hline \multicolumn{2}{|c|}{ Tiêu chí đánh giá } & Tần suất & Tỷ lệ (\%) & \multicolumn{2}{|c|}{ Tiêu chí đánh giá } & Tần suất & Tỷ lệ (\%) \\
\hline \multirow{3}{*}{$\begin{array}{l}\text { Số năm kinh } \\
\text { nghiệm }\end{array}$} & Dưới 3 năm & 25 & $22,8 \%$ & \multirow{3}{*}{ Đơn vị } & Chủ đầu tư, B.QLDA & 67 & $60,9 \%$ \\
\hline & Từ 3 - 5 năm & 26 & $23,7 \%$ & & Tư vấn thiết kế & 19 & $17,3 \%$ \\
\hline & Trên 10 năm & 32 & $29 \%$ & & Nhà thầu thi công & 19 & $17,3 \%$ \\
\hline \multirow{3}{*}{$\begin{array}{l}\text { Dự án tham gia } \\
\text { (nhiều lựa } \\
\text { chọn) }\end{array}$} & Chung cư & 95 & $41,1 \%$ & \multirow{3}{*}{$\begin{array}{l}\text { Quy mô dự } \\
\text { án }\end{array}$} & Dưới 100 tỷ & 7 & $6,4 \%$ \\
\hline & \multirow{2}{*}{$\begin{array}{l}\text { Trung tâm thương mại, } \\
\text { Phức hợp }\end{array}$} & \multirow{2}{*}{69} & \multirow{2}{*}{$29,9 \%$} & & Từ 200 tỷ đến 500 tỷ & 26 & $23,6 \%$ \\
\hline & & & & & Trên 500 tỷ & 61 & $55,5 \%$ \\
\hline
\end{tabular}

\section{2. Đánh giá độ tin cậy của thang đo}

Nghiên cứu tiến hành kiểm tra hệ số Cronbach's Alpha để đánh giá độ tin cậy của thang đo. Để tăng giá trị khi đánh giá rủi ro, loại 02 biến E3 (do giá trị trung bình của mức độ tác động nhỏ hơn 3) và E5 (do khả năng xảy ra có giá trị trung bình nhỏ hơn 2), tổng số nhân tố còn lại là 26. 
Bảng 4.

Kết quả kiểm định thang đo Cronbach's Alpha.

\begin{tabular}{|c|c|c|c|c|c|}
\hline \multicolumn{3}{|c|}{ CHİ TIÊU CHI PHÍ } & \multicolumn{3}{|c|}{ CHİ TIÊU TIẾN Độ } \\
\hline Biến & $\begin{array}{l}\text { Corrected Item-Total } \\
\text { Correlation }\end{array}$ & $\begin{array}{l}\text { Cronbach's Alpha } \\
\text { if Item Deleted }\end{array}$ & Biến & $\begin{array}{c}\text { Corrected Item-Total } \\
\text { Correlation }\end{array}$ & $\begin{array}{c}\text { Cronbach's Alpha if Item } \\
\text { Deleted }\end{array}$ \\
\hline \multicolumn{3}{|c|}{ 1. Tài chính và Kinh tế: $\alpha=0,781$} & \multicolumn{3}{|c|}{ 1. Tài chính và Kinh tế: $\alpha=0,818$} \\
\hline CPA1 & 0,305 & 0,783 & TDA1 & 0,582 & 0,791 \\
\hline CPA2 & 0,303 & 0,784 & TDA2 & 0,546 & 0,796 \\
\hline CPA3 & 0,519 & 0,753 & TDA3 & 0,387 & 0,816 \\
\hline CPA4 & 0,382 & 0,772 & TDA4 & 0,502 & 0,802 \\
\hline CPA5 & 0,662 & 0,726 & TDA5 & 0,629 & 0,784 \\
\hline CPA6 & 0,547 & 0,746 & TDA6 & 0,594 & 0,789 \\
\hline CPA7 & 0,546 & 0,746 & TDA7 & 0,476 & 0,806 \\
\hline CPA8 & 0,604 & 0,735 & TDA8 & 0,572 & 0,793 \\
\hline \multicolumn{3}{|c|}{ 2. Chính sách và Pháp luật: $\alpha=0,733$} & \multicolumn{3}{|c|}{ 2. Chính sách và Pháp luật: $\alpha=0,681$} \\
\hline CPB1 & 0,496 & 0,686 & TDB1 & 0,507 & 0,597 \\
\hline СРB2 & 0,609 & 0,651 & TDB2 & 0,460 & 0,619 \\
\hline СРB3 & 0,426 & 0,712 & TDB3 & 0,492 & 0,614 \\
\hline СРВ4 & 0,568 & 0,656 & TDB4 & 0,317 & 0,693 \\
\hline CPB5 & 0,404 & 0,726 & TDB5 & 0,440 & 0,628 \\
\hline \multicolumn{3}{|c|}{ 3. Kỹ thuật và Công nghệ: $\alpha=0,774$} & \multicolumn{3}{|c|}{ 3. Kỹ thuật và Công nghệ: $\alpha=0,808$} \\
\hline CPC1 & 0,534 & 0,737 & TDC1 & 0,538 & 0,789 \\
\hline CPC2 & 0,553 & 0,731 & TDC2 & 0,535 & 0,789 \\
\hline CPC3 & 0,533 & 0,737 & TDC3 & 0,681 & 0,745 \\
\hline CPC4 & 0,470 & 0,758 & TDC4 & 0,567 & 0,779 \\
\hline CPC5 & 0,647 & 0,696 & TDC5 & 0,658 & 0,751 \\
\hline \multicolumn{3}{|c|}{ 4. Tổ chức và Quản lý: $\alpha=0,823$} & \multicolumn{3}{|c|}{ 4. Tổ chức và Quản lý: $\alpha=0,824$} \\
\hline CPD1 & 0,543 & 0,810 & TDD1 & 0,514 & 0,817 \\
\hline CPD2 & 0,595 & 0,795 & TDD2 & 0,676 & 0,771 \\
\hline CPD3 & 0,675 & 0,776 & TDD3 & 0,705 & 0,764 \\
\hline CPD4 & 0,662 & 0,776 & TDD4 & 0,621 & 0,788 \\
\hline CPD5 & 0,630 & 0,785 & TDD5 & 0,579 & 0,800 \\
\hline \multicolumn{3}{|c|}{ 5. Môi trường và Xã hội: $\alpha=0,653$} & \multicolumn{3}{|c|}{ 5. Môi trường và Xã hội: $\alpha=0,763$} \\
\hline CPE1 & 0,493 & 0,522 & TDE1 & 0,602 & 0,677 \\
\hline CPE2 & 0,542 & 0,444 & TDE2 & 0,656 & 0,610 \\
\hline CPE4 & 0,377 & 0,662 & TDE4 & 0,535 & 0,747 \\
\hline
\end{tabular}

Kết quả kiểm định thang đo cho thấy:

- Các biến quan sát đều có hệ số tương quan tổng biến (Corrected Item-Total Correlation) là phù hợp ( đều $\geq 0,3$ ) [9].

- Hệ số Cronbach's Alpha phần lớn nằm trong khoảng $0,60 \leq \alpha \leq$ 1,00 , hệ số tổng >0,6: Thang đo lường tốt. Nên đạt yêu cầu về độ tin cậy [10].

- Từ kết quả kiểm tra hệ số Cronbach's Alpha cho thấy dữ liệu có độ tin cậy cao và tiếp tục được sử dụng cho các phân tích tiếp theo.

\subsection{Kết quả phân tích EFA}

Trong nghiên cứu, số lượng các biến thu về là khá nhiều và chúng có mối liên hệ với nhau. Với mục đích là tìm ra số lượng các nhân tố ảnh hưởng đến 1 nhóm và mối quan hệ giữa những mối nhân tố với biến đo lường. Vì thế, việc sử dụng phân tích nhân tố khám phá (EFA) để phân tích số liệu trong nghiên cứu này là hợp lý.

\subsubsection{Kiểm định EFA cho nhóm tiêu chí Chi phí}


Kết quả sau khi tiến hành phân tích EFA lần 1 , các biến CPA4 và CPE4 nằm trong diện bị loại vì có hệ số tải < 0,5 (biến này không được tải lên ở nhân tố nào). Tiến hành phân tích EFA lần 2.

\section{Bảng 5.}

Hệ số KMO and Bartlett's Test lần 2 nhóm tiêu chí Chi phí.

\begin{tabular}{|c|c|c|}
\hline \multicolumn{3}{|c|}{ KMO and Bartlett's Test } \\
\hline \multicolumn{2}{|c|}{ Kaiser-Meyer-Olkin Measure of Sampling Adequacy. } & 0,715 \\
\hline \multirow{3}{*}{ Bartlett's Test of Sphericity } & Approx. Chi-Square & 1008,117 \\
\hline & df & 276 \\
\hline & Sig. & 0,000 \\
\hline
\end{tabular}

\section{Bảng 6.}

Ma trận xoay lần 2 nhóm tiêu chí Chi phí.

\begin{tabular}{|c|c|c|c|c|c|c|}
\hline \multirow{2}{*}{ Nhân tố } & \multicolumn{6}{|c|}{ Nhóm } \\
\hline & 1 & 2 & 3 & 4 & 5 & 6 \\
\hline CPA8 & 0,819 & & & & & \\
\hline CPA7 & 0,730 & & & & & \\
\hline CPA5 & 0,725 & & & & & \\
\hline CPA3 & 0,688 & & & & & \\
\hline CPA6 & 0,537 & & & & & \\
\hline CPD1 & & 0,812 & & & & \\
\hline CPD4 & & 0,685 & & & & \\
\hline CPD3 & & 0,656 & & & & \\
\hline CPD2 & & 0,647 & & & & \\
\hline CPD5 & & 0,537 & & & & \\
\hline CPC5 & & & 0,781 & & & \\
\hline CPC3 & & & 0,709 & & & \\
\hline CPC1 & & & 0,672 & & & \\
\hline CPC2 & & & 0,636 & & & \\
\hline CPC4 & & & 0,606 & & & \\
\hline CPB2 & & & & 0,804 & & \\
\hline CPB4 & & & & 0,727 & & \\
\hline CPB1 & & & & 0,683 & & \\
\hline СРB3 & & & & 0,600 & & \\
\hline CPB5 & & & & 0,531 & & \\
\hline CPE1 & & & & & 0,818 & \\
\hline CPE2 & & & & & 0,810 & \\
\hline CPA2 & & & & & & 0,770 \\
\hline CPA1 & & & & & & 0,768 \\
\hline $\begin{array}{c}\text { Eigenv } \\
\text { alues }\end{array}$ & 5,236 & 3,461 & 2,118 & 1,552 & 1,251 & 1,173 \\
\hline $\begin{array}{c}\text { \% biến } \\
\text { thiên }\end{array}$ & 21,818 & 14,423 & 8,823 & 6,468 & 5,213 & 4,886 \\
\hline $\begin{array}{c}\text { \% Tích } \\
\text { lũy }\end{array}$ & 21,818 & 36,240 & 45,064 & 51,532 & 56,745 & 61,631 \\
\hline
\end{tabular}

Kết quả sau khi tiến hành phân tích EFA lần 2:
- Chỉ số Kaiser-Meyer-Olkin (KMO): KMO = 0,715 > 0,5.

- Bartlett's test: Sig. $=0,00<0,05$.

- Hệ số Eigenvalue $=1,173>1$ và trích được 6 nhân tố mang ý nghĩa tóm tắt được thông tin là tốt nhất.

- Percentage of variance $=61,631 \%>50 \%$ cho thấy mô hình EFA là phù hợp. Có nghĩa là các nhân tố trích cô đọng được $61,631 \%$ biến thiên của các biến quan sát.

- Kết quả ma trận xoay lần 02, 24 biến quan sát được gom thành 06 nhân tố và các biến quan sát điều có hệ số tải nhân tố Factor Loading $>0,5$.

Kết quả phân tích EFA lần 2 chỉ ra rằng 06 nhóm yếu tố có liên quan Tài chính, Kinh tế, Chính sách, Pháp luật, Kỹ thuật, Công nghệ, Tổ chức, Quản lý, Môi trường và Xã hội. Dựa trên kết quả, có thể đặt tên các nhóm lại như sau: Nhóm 1 - Tài chính, Nhóm 2 - Tổ chức và Quản lý, Nhóm 3 - Kỹ thuật và Công nghệ, Nhóm 4 - Chính sách và Pháp luật, Nhóm 5 - Môi trường và xã hội, Nhóm 6 - Kinh tế.

\subsubsection{Kiểm định EFA cho nhóm tiêu chí Tiến độ}

Kết quả sau khi tiến hành phân tích EFA lần 1, loại biến TDA4 (có hệ số tải nhỏ hơn 0,5 , do các biến ngày không tải lên ở nhân tố nào) và TDE4 (tải lên cả 2 nhân tố 5 và 7). Kết quả sau khi tiến hành phân tích EFA lần 2, loại biến TDB5 (nằm tách biệt và duy nhất chỉ ở 1 nhân số 7). Kết quả sau khi tiến hành phân tích EFA lần 3, loại biến TDA6 (tải lên cả nhân tố 2, 3). Tiến hành phân tích EFA lần 4.

Bảng 7.

Hệ số KMO and Bartlett's Test lần 4 nhóm tiêu chí Tiến độ.

\begin{tabular}{|c|c|c|}
\hline \multicolumn{3}{|c|}{ KMO and Bartlett's Test } \\
\hline \multicolumn{2}{|c|}{ Kaiser-Meyer-Olkin Measure of Sampling Adequacy. } & 0,725 \\
\hline \multirow{3}{*}{ Bartlett's Test of Sphericity } & $\begin{array}{l}\text { Approx. Chi- } \\
\text { Square }\end{array}$ & 1006,862 \\
\hline & df & 231 \\
\hline & Sig. & 0,000 \\
\hline
\end{tabular}

Bảng 8.

Ma trận xoay lần 4 nhóm tiêu chí Tiến độ.

\begin{tabular}{|c|c|c|c|c|c|c|}
\hline \multirow{2}{*}{ Nhân tố } & \multicolumn{7}{|c|}{ Nhóm } \\
\hline & 1 & 2 & 3 & 4 & 5 & 6 \\
\hline TDC4 & 0,753 & & & & & \\
\hline TDC5 & 0,748 & & & & & \\
\hline TDC2 & 0,736 & & & & & \\
\hline TDC3 & 0,654 & & & & & \\
\hline TDD1 & 0,625 & & & & & \\
\hline TDD2 & 0,559 & & & & & \\
\hline TDD3 & 0,542 & & & & & \\
\hline TDA1 & & 0,804 & & & & \\
\hline
\end{tabular}




\begin{tabular}{|c|c|c|c|c|c|c|}
\hline \multirow{2}{*}{ Nhân tố } & \multicolumn{5}{|c|}{ Nhóm } \\
\hline & 1 & 2 & 3 & 4 & 5 & 6 \\
\hline TDA2 & & 0,777 & & & & \\
\hline TDA5 & & 0,613 & & & & \\
\hline TDD4 & & & 0,751 & & & \\
\hline TDD5 & & & 0,748 & & & \\
\hline TDC1 & & & 0,640 & & & \\
\hline TDB2 & & & & 0,804 & & \\
\hline TDB1 & & & & 0,762 & & \\
\hline TDB3 & & & & 0,630 & & \\
\hline TDA3 & & & & 0,560 & & \\
\hline TDB4 & & & & & 0,793 & \\
\hline TDA7 & & & & & 0,769 & \\
\hline TDA8 & & & & & 0,711 & \\
\hline TDE1 & & & & & & 0,856 \\
\hline TDE2 & & & & & & 0,782 \\
\hline $\begin{array}{c}\text { Eigenval } \\
\text { ues }\end{array}$ & 5,146 & 3,574 & 1,856 & 1,500 & 1,261 & 1,096 \\
\hline $\begin{array}{c}\text { \% biến } \\
\text { thiên }\end{array}$ & 23,391 & 16,244 & 8,435 & 6,819 & 5,733 & 4,981 \\
\hline $\begin{array}{c}\text { \% Tích } \\
\text { lũy }\end{array}$ & 23,391 & 39,635 & 48,070 & 54,890 & 60,622 & 65,603 \\
\hline
\end{tabular}

Kết quả sau khi tiến hành phân tích EFA lần 4:

- Chỉ số Kaiser-Meyer-Olkin (KMO): KMO = 0,725 > 0,5

- Bartlett's test: Sig. $=0,00<0,05$.

- Hệ số Eigenvalue $=1,096>1$ và trích được 6 nhân tố mang ý nghĩa tóm tắt được thông tin là tốt nhất.

- Percentage of variance $=65,603 \%>50 \%$ cho thấy mô hình EFA là phù hợp. Có nghĩa là các nhân tố trích cô đọng được 65,603 \% biến thiên của các biến quan sát.

- Kết quả ma trận xoay lần 04, 22 biến quan sát được gom thành 06 nhân tố và các biến quan sát điều có hệ số tải nhân tố Factor Loading $>0,5$.

Kết quả phân tích EFA lần 4 chỉ ra rằng 06 nhóm yếu tố có liên quan Tài chính, Kinh tế, Chính sách, Pháp luật, Kỹ thuật, Công nghệ, Tổ chức, Quản lý, Môi trường và Xã hội. Dựa trên kết quả, có thể đặt tên các nhóm lại như sau: Nhóm 1 - Kỹ thuật và Tổ chức, Nhóm 2 Kinh tế, Nhóm 3 - Quản lý, Nhóm 4 - Chính sách và Pháp luật, Nhóm 5 - Tài chính, Nhóm 6 - Môi trường và xã hội.

Các đối tượng khảo sát đánh giá nhân tố liên quan đến Kỹ thuật/Tổ chức đóng vai trò ảnh hưởng nhất trong việc giải thích sự biến thiên của dữ liệu là $23,391 \%$. Điều này cho thấy các rủi về Kỹ thuật/Tổ chức có mức độ tác động lớn đến tiến độ trong giai đoạn chuẩn bị dự án. Yêu cầu Chủ đầu tư phải chú trọng việc lựa chọn kỹ thuật và công nghệ phù hợp với dự án, chú trọng năng lực các đơn vị tham gia và phải xây dựng cơ cấu tổ chức phù hợp để điều hành và quản lý.

Đóng vai trò ảnh hưởng thứ hai trong việc giải thích sự biến thiên của dữ liệu với 16,244 \% là nhân tố liên quan đến Kinh tế. Điều này cho thấy các rủi ro liên quan đến kinh tế luôn luôn tồn tại và ảnh hưởng đến tiến độ. Cần chú trọng công tác nghiên cứu kinh doanh, thị trường để kiểm soát tốt các hoạt động quản lý rủi ro.

Tiếp theo là nhân tố liên quan đến Quản lý, được các đối tượng khảo sát nhận định đóng vai trò ảnh hưởng thứ ba với giá trị giải thích sự biến thiên của dữ liệu là $8,435 \%$. Việc quản lý tốt các hợp đồng và giao tiếp thông tin hiệu quả giữa các bên luôn luôn là chìa khóa dẫn đến thành công cho dự án.

Tiếp đến đóng vai trò ảnh hưởng thứ tư là nhân tố liên quan đến Chính sách/Pháp luật với giá trị giải thích sự biến thiên của dữ liệu là $6,819 \%$. Thực tế, các rủi ro liên quan đến chính sách luôn luôn tiềm ẩn những rủi ro làm ảnh hưởng đến tiến độ trong giai đoạn chuẩn bị dự án, các nhân tố này cần được quan tâm nhiều hơn vì có mức độ ảnh hưởng đáng kể.

Ngoài ra, nhân tố liên quan đến Tài chính giải thích được sự biến thiên của dữ liệu là 5,733 \% đóng vai trò ảnh hưởng thứ năm. Tương tự như Kinh tế, việc phân tích và quản lý luôn luôn là bài toán quan trọng, yêu cầu Chủ đầu tư cần quan tâm thực hiện và giảm thiểu các rủi ro không đáng có.

Cuối cùng, nhân tố Môi trường/Xã hội đóng vai trò ảnh hưởng thứ sáu với giải thích được sự biến thiên của dữ liệu là 4,981 \%. Bên cạnh các yếu tố rủi ro quan trọng trong việc đánh giá tiến độ dự án trong giai đoạn chuẩn bị, để đảm bảo thành công chung cho dự án thì Chủ đầu tư cũng cần xem xét và đánh giá các nhóm rủi ro liên quan đến Môi trường và Xã hội, các nhân tố này cần được quan tâm và xem xét.

\subsection{Kết quả xếp hạng các nhân tố theo giá trị trung bình}

Việc đánh giá xếp hạng các rủi ro là sự kết hợp giữa Xác suất xảy ra và Mức độ ảnh hưởng: $(\mathrm{R})=$ (Khả năng xảy ra) $\mathrm{x}$ (Mức độ ảnh huởng) $=\mathrm{P} \times \mathrm{I}$, kết quả cho thấy bảng xếp hạng của 5 nhân tố cao nhất từ bảng xếp hạng giá trị trung bình có giá trị từ 12,55 đến 14,93.

\section{Bảng 9.}

Xếp hạng của 5 nhân tố rủi ro cao nhất giai đoạn chuẩn bị dự án.

\begin{tabular}{|c|c|c|c|c|}
\hline Nhân tố & $\begin{array}{c}\text { Khả năng } \\
\text { xảy ra }\end{array}$ & $\begin{array}{c}\text { Mức độ tác } \\
\text { động }\end{array}$ & $\begin{array}{c}\text { Giá trị trung } \\
\text { bình tổng }\end{array}$ & Xếp hạng \\
\hline A3 & 3,35 & 4,45 & 14,93 & 1 \\
\hline B5 & 3,54 & 4,08 & 14,42 & 2 \\
\hline B3 & 3,41 & 4,15 & 14,15 & 3 \\
\hline B1 & 3,29 & 4,21 & 13,85 & 4 \\
\hline D4 & 3,54 & 3,55 & 12,55 & 5 \\
\hline
\end{tabular}


Căn cứ theo kết quả khảo sát đại trà, 05 nhân tố có xếp hạng cao nhất lần lượt là: A3-B5-B3-B1-D4, đây đều là những nhân tố rủi ro gây ảnh hưởng lớn đến dự án trong giai đoạn chuẩn bị dự án.

Xếp hạng thứ nhất là thiếu hụt nguồn vốn, khả năng huy động vốn không tốt (A3):

- Lựa chọn phương án và phân bổ nguồn vốn chưa phù hợp và thiếu đối tác chiến lược hợp tác lâu dài. Việc huy động nguồn vốn là yếu tố rất quan trọng, ảnh hưởng trực tiếp đến hiệu quả đầu tư xây dựng của Chủ đầu tư. Trong bối cảnh ngân hàng thắt chặt tín dụng, lãi suất tăng cao thì giải pháp huy động vốn khác ngoài ngân hàng quyết định đến tính khả thi của dự án. Các giải pháp đó có thể là phát hành cổ phiếu, trái phiếu...

- Không xác định đúng nguồn vốn lưu động của công ty sẽ gây ra tình trạng thiếu vốn hoặc sẽ làm gián đoạn hoạt động đầu tư. Hầu hết các dự án Bất động sản đều có cơ cấu vốn vay ngân hàng. Khi thị trường biến động, tỷ lệ huy động nguồn vốn từ các nhà đầu tư là rất khó khăn, lãi vay ngân hàng tăng cao dẫn đến không kiểm soát được nguồ vốn, dẫn đến dự án gặp thất bại.

- Thực tế, có rất nhiều doanh nghiệp xây dựng vừa và nhỏ phải cố sức chi trả các chi phí, việc lấy tiền của dự án này để đầu tư dự án khác là ví dụ điển hình, đi kèm với đó là việc thiết hụt nguồn vốn (thiếu hụt tiền mặt liên tục) thực tại tại chính dự án.

Xếp hạng thứ hai là khó khăn khi xin cấp phép xây dựng (B5):

- Các thủ tục, quy định liên quan nhưng không rõ ràng, các thủ tục chồng chéo gây khó khăn trong quá trình xin phép xây dựng. Từ đó dẫn đến thực trạng là Cơ quan quản lý cấp giấy phép xây dựng phải yêu cầu Chủ đầu tư gửi các văn bản và có phản hồi từ các Cơ quan Quản lý có liên quan trước khi nộp hồ sơ xin phép xây dựng. Thực tế kinh doanh Bất động sản bị chi phối và quản lý chặt bởi các Luật, Nghị định, Thông tư và các văn bản quy phạm pháp luật khác. Trong đó có rất nhiều điều khoản của Luật, Nghị định thường chồng chéo, mâu thuẩn làm cho Chủ đầu tư gặp nhiều khó khăn. Ảnh hưởng trực tiếp đến tiến độ chuẩn bị dự án.

- Khối lượng hồ sơ chuẩn bị lớn, huy động nguồn nhân lực chuẩn bị, thời gian kéo dài. Thủ tục hành chính nhiều khê, thông qua nhiều Cơ quan ban ngành, đòi hỏi yêu cầu phải bố trí nhân sự chuyên biệt để thực hiện công tác.

- Theo Sở Xây dựng Tp.HCM thì UBND Tp.HCM đã có văn bản gửi Bộ Xây dựng đề nghị sớm có thông tư, hướng dẫn chi tiết để tháo gỡ các vướng mắc, tuy nhiên đến bao giờ nhận được lại là là dấu hỏi lớn cần giải đáp.

Xếp hạng thứ ba là điều chỉnh và phê duyệt hồ sơ đầu tư, hồ sơ thiết kế kéo dài, nhiều thủ tục phát sinh từ CQQL Nhà nước (B3):

- Thời gian điều chỉnh hồ sơ Thiết kế cơ sở, Thiết kế kỹ thuật, Thiết kế PCCC kéo dài. Nhiều ý kiến góp ý phải tiến hành làm đi làm lại nhiều lần. Cơ quan Quản lý Nhà nước làm việc quan liêu, thiếu trách nhiệm. Nhiều thủ tục phát sinh ảnh hưởng đến công tác xin cấp giấy phép xây dựng và các văn bản chấp thuận khác. Bên cạnh đó là các vấn đề tiêu cực khác trong quá trình xin phép phê duyệt dự án, gây lãng phí thời gian và chi phí.

- Thực tế thời gian thực hiện dự án thường khá dài nên tình hình Bất động sản sẽ biến động. Tùy theo tình hình thực tế của thị trường và khả năng tài chính mà Chủ đầu tư sẽ có xu hướng điều chỉnh, thay đổi quy mô, công năng, công nghệ sử dụng trong dự án để thu lại lợi nhuận cao nhất. Khi đó việc điều chỉnh thiết kế sẽ làm kéo dài thời gian thực hiện dự án. Bên cạnh đó, theo thời gian thì tác động của lạm phát, trượt giá, những biến động khác sẽ làm ảnh hưởng đến hiệu quả đầu tư.

Xếp hạng thứ tư là vướng công tác đền bù, giải phóng mặt bằng (B1):

- Vướng công tác đền bù, giải phóng mặt bằng thường tập trung ở các vấn đề: Nguồn gốc đất sử dụng, giá bồi thường chênh lệch so với thực tế, thủ tục không minh bạch... Nhiều trường hợp gây bức xúc, dẫn đến các hành vi gây cản trở và không chịu di dời, ảnh hưởng trực tiếp đến kế hoạch chuẩn bị, kéo dài tiến độ thi công.

- Đền bù và giải pháp mặt bằng là một khâu quan trọng trong công tác triển khai thực hiện dự án, quyết định đến tính khả thi của dự án đầu tư xây dựng. Việc gặp vướng mắc trong đền bù giải phòng mặt bằng sẽ ảnh hưởng trực tiếp đến tiến độ thực hiện dự án, kéo dài thời gian và phát sinh chi phí. Nếu dự án không được giải phóng mặt bằng đúng tiến độ, Chủ đầu tư sẽ gặp trở ngại lớn trong việc đầu tư vào dự án.

- Thực tế, có rất nhiều dự án sai phạm vì vướng công tác giải phóng mặt bằng mà dự án kéo dài điển hình như các dự án tại Khu đô thị mới Thủ Thiêm, Tp.HCM. Mở rộng xa hơn là chuỗi các dự án sai phạm tại tỉnh Khánh Hòa năm 2016 hay dự án đường giao thông bao quanh khu tưởng niệm danh nhân Chu Văn An, Tp. Hà Nội năm 2017.

Xếp hạng thứ năm là các bên phối hợp, giao tiếp thông tin không hiệu quả (D4):

- Đặc điểm của dự án đầu tư xây dựng là thực hiện trong thời gian dài, việc có quá nhiều đơn vị tư vấn, nhà thầu, nhà cung cấp cùng tham gia thực hiện dự án sẽ gây ra tình trạng hỗn loạn thông tin, việc trao đổi và giao tiếp thường xuyên là yêu cầu bắt buộc. Các cuộc họp phối hợp giữa các bên cần phải thường xuyên được tổ chức và kiểm soát.

- Một dự án thành công thì phải có sự kết hợp từ các bên tham gia trong dự án, phối hợp thông tin nhịp nhàng giữa các bên. Mức độ hợp tác, phối hợp phần nào sẽ đánh giá được mức độ thành công chung của dự án. Phối hợp tốt, giao tiếp hiệu quả sẽ là nền phát hướng đến thành công chung của dự án. 
- Trong thực tế, các Chủ đầu tư không chỉ giao tiếp bằng văn bản mà họ còn phải trao đổi trực tiếp, bàn bạc với các đối tác, nhà thầu. Việc tiếp nhận thông tin nguồn thông tin không chính thức, chưa phối hợp với các bên trước khi ra quyết định sẽ làm ảnh hưởng đến hiệu quả của dự án. Các thông tin phải được cập nhật liên tục và kịp thời từ các bên khác nhau. Hiệu quả các công việc trong vòng đời dự án sẽ phụ thuộc và nhiều yếu tố, một trong những yếu tố không thể thiếu và phải yêu cầu xuyên suốt trong dự án đó là: "Tuân thủ nguyên tắc phối hợp" để đảm bảo hiệu quả thông tin khi giao tiếp.

\section{Kết luận và kiến nghị}

Việc xác định và phân tích các nhân tố rủi ro gặp phải của Chủ đầu tư trong giai đoạn chuẩn bị thực hiện đã đóng góp một số những kiến thức cơ bản về rủi ro có thể xảy trong trong vòng đời của dự án đầu tư xây dựng. Các nhóm yếu tố rủi ro Chủ đầu tư có thể gặp phải đã được tổng hợp và cô đọng thành các nhóm chính. Nghiên cứu đã chỉ ra được 26 nhân tố rủi ro, các nhân tố này được sắp xếp thành 05 nhóm chính và gây ảnh hưởng trực tiếp đến dự án. Ngoài ra nghiên cứu cũng đã phân tích và đánh giá mức độ rủi ro của dự án dựa trên 02 tiêu chí chính (đặc thù của giai đoạn chuẩn bị): Chi phí và Tiến độ.

Việc xác định, phân tích và đánh giá rủi ro sẽ giúp cho Chủ đầu tư nhìn nhận tổng quan hơn về cơ hội và thách thức khi tham gia thực hiện dự án. Trên cơ sở đó, phát huy tối đa được điểm mạnh, hạn chế điểm yếu nhằm nâng cao tối đa hiệu quả của dự án, duy trì tiềm lực và hình ảnh Công ty, tạo dựng mức độ uy tín và danh tiếng trong thị trường Bất động sản tại Tp.HCM nói riêng và tại Việt Nam hoặc trên thế giới nói chung.

\section{Tài liệu tham khảo}

[1]. AS/NZS 4360-1999 - Standards Australia - Risk management.

[2]. ICE - Institution of Civil Engineers. Ramp book - Risk analysis and Management for projects, Thomas Telford.

[3]. PMBOK Guide - Sixth Edition + Agile practice guide.

[4]. Tạp chí người xây dựng (Số 11 \& 12 năm 2018). Tổng quan các nghiên cứu về quản lý rủi ro trong xây dựng.

[5]. Fangfang $\mathrm{Wen}^{1}$, Ling $\mathrm{Li}^{2}$ (2013). Based on AHP-fuzzy comprehensive evaluation method of real estate investment risk research.

[6]. Bayu Aditya Firmansyah ${ }^{1}$, Alin Veronika ${ }^{2}$, Bambang Trigunarsyah ${ }^{3}$ (2006). Risk analysis in feasibility study of building construction project: Case study Pt. Perusahaan gas negara Indonesia.

[7]. Nguyễn Quốc Toản (2019). Quản lý rủi ro: Bài học kinh nghiệm và giải pháp trong giai đoạn chuẩn bị dự án (Khảo sát tại Ban quản lý dự án Thành phố Uông Bí, Quảng Ninh).
[8]. Lê Văn Long (2006), Một số vấn đề quản lý rủi ro trong dự án đầu tư xây dựng công trình,Tạp chí Kinh tế Xây dựng, số 4/2006.

[9]. Nunnally, J. (1978), Psychometric Theory, New York, McGrawHill.

[10]. Hoàng Trọng, Chu Nguyễn Mộng Ngọc (2008), Phân tích dữ liệu nghiên cứu với SPSS, NXB Hồng Đức.

[11]. Luật Xây dựng số 50/2014/QH13 ban hành ngày 18 tháng 06 năm 2014.

[12]. Nghị định số: 15/2021/NĐ-CP ngày 03 tháng 3 năm 2021 về quản lý dự án đầu tư xây dựng.

[13]. Roger Flanagan, George Norman (1993). Risk Management and Construction, Blackwell Scientific.

[14]. Nigel J. Smith, Tony Merna, Paul Jobling (2006). Managing Risk in Construction Projects, Second edition-Wiley-Blackwell.

[15]. John Raftery (1993). Risk Analysis in Project Management, E\&FN Spon. 